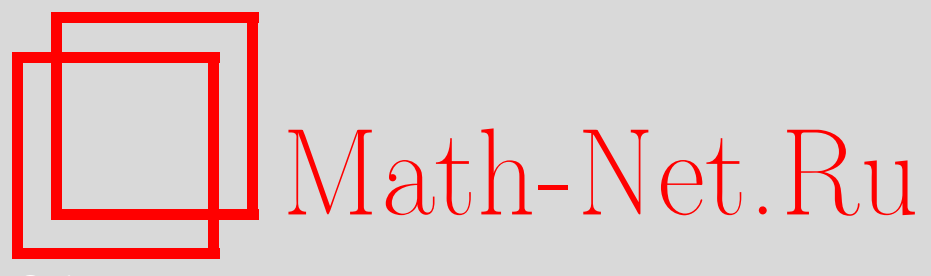

Г. А. Васильев, В. М. Хаметов, Е. А. Шелемех, Об условиях дискретности экстремальных вероятностных мер (конечномерный случай), Матем. заметки, 2013, том 94, выпуск 6, 944-948

DOI: https://doi.org/10.4213/mzm10368

Использование Общероссийского математического портала Math-Net.Ru подразумевает, что вы прочитали и согласны с пользовательским соглашением http://www . mathnet.ru/rus/agreement

Параметры загрузки:

IP: 3.91 .87 .62

26 апреля 2023 г., 16:13:13

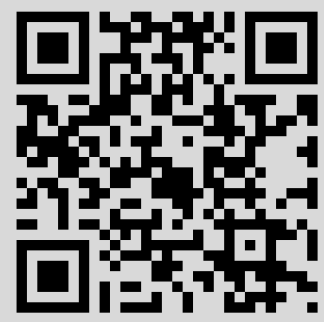




\section{Об условиях дискретности экстремальных вероятностных мер (конечномерный случай)}

\section{Г. А. Васильев, В. М. Хаметов, Е. А. Шелемех}

В данном сообщении устанавливаются условия дискретности экстремальной вероятностной меры на конечномерных пространствах. Эта проблема существует в теории Шоке [1]-[6], стохастической финансовой математике [7]-[9], при построении примеров решения задачи Монжа-Канторовича [10].

Доказательство основного результата статьи не опирается на вышеупомянутые работы.

1. Напомним некоторые обозначения и определения. Пусть $(E, \mathscr{E})$ - измеримое пространство, а $M(E)$ - множество вероятностных мер на $(E, \mathscr{E})$. Пусть $f: E \rightarrow \mathbb{R}^{+}$- любая $\mathscr{E}$-измеримая положительная ограниченная функция. Будем использовать следующие обозначения:

i) $\operatorname{extr} A$ - множество крайних точек множества $A$;

ii) $I^{\mu} \triangleq|\operatorname{supp} \mu|, \mu \in M(E)$.

2. Для формулировки основного утверждения работы нам потребуются определение и вспомогательное утверждение.

ОПредЕлениЕ 1 . Вероятностную меру $\mu^{*}$ на $(E, \mathscr{E})$ будем называть экстремальной относительно множества вероятностных мер $\mathfrak{R} \subseteq M(E)$, если для любой $\mathscr{E}$-измеримой ограниченной функции $f: E \rightarrow \mathbb{R}^{+}$справедливо равенство

$$
\sup _{\mu \in \mathfrak{R}} \int_{E} f(x) \mu(d x)=\int_{E} f(x) \mu^{*}(d x) .
$$

Предложение 1. Экстремальная относительно множества $\mathfrak{R} \subseteq M(E)$ вероятностная мера $\mu^{*}$ существует тогда и только тогда, когда $\mathfrak{R}$ - слабо относительно компактное множество.

ЗАмечАниЕ 1. Достаточность условия в предложении 1 следует из слабой относительной компактности множества $\mathfrak{R}$, определения верхней грани и теоремы Данфорда-Петтиса. Доказательство этого утверждения почти дословно повторяет доказательство теоремы 5 работы [9]. Необходимость - очевидна.

ЗАмечание 2. Пусть $\mathfrak{R} \subseteq M(E)$ такое, что $\sup _{\mu \in \mathfrak{R}} \int_{E}|x| \mu(d x)<\infty$. Известно [11; $\S 2$, гл. III], что в этом случае $\mathfrak{R}$ - слабо относительно компактное множество и существуют экстремальная относительно него вероятностная мера $\mu^{*}$ и конечный интеграл Лебега $m^{*} \triangleq$ $\int_{E} x \mu^{*}(d x)$.

3. Пусть $D \triangleq\left\{\gamma \in \mathbb{R}^{d}: \int_{E} e^{f(x)-\left(\gamma, x-m^{*}\right)} \mu^{*}(d x)<\infty\right\}$. Очевидно, что $D \neq \varnothing$.

Следующее утверждение - это основной результат данной работы.

Теорема 1. Справедливы следующие утверждения.

1) Пусть $E=\mathbb{R}^{d}, d<\infty$. Экстремальная относительно множества $\mathfrak{R}$ вероятностная мера $\mu^{*}$ - дискретна тогда и толъко тогда, когда существует $\gamma^{*} \in D$ такой, что выполняется неравенство:

$$
\int_{E} e^{f(x)-\left(\gamma^{*}, x-m^{*}\right)} \mu^{*}(d x) \leqslant \int_{E} e^{f(x)-\left(\gamma, x-m^{*}\right)} \mu^{*}(d x),
$$

где $\gamma$ - любой из $D$, при этом $I^{\mu^{*}} \leqslant d+1$.

DOI: $10.4213 / \mathrm{mzm} 10368$ 
2) Пусть $E-d$-мерный компакт, $d<\infty$. Тогда существует вероятностная мера $\mu^{*} \in M(E)$ - дискретная, $I^{\mu^{*}} \leqslant d+1$, такая, что справедливы равенства

$$
\sup _{\mu \in \Re} \int_{E} f(x) \mu(d x)=\int_{E} f(x) \mu^{*}(d x)=\sum_{i=1}^{I^{\mu^{*}}} c_{i} f\left(x_{i}\right),
$$

где $x_{i} \in \operatorname{extr} E, 1 \leqslant i \leqslant I^{\mu^{*}}$, причем $c_{i} \triangleq \mu^{*}\left(\left\{x_{i}\right\}\right)>0 u \sum_{i=1}^{I^{\mu^{*}}} c_{i} x_{i}=m^{*}, \sum_{i=1}^{I^{\mu^{*}}} c_{i}=1$.

4. Данный пункт посвящен доказательству теоремы 1 , которое опирается на решение рассматриваемой ниже вспомогательной задачи.

4.1. Рассмотрим вспомогательную задачу

$$
\int_{E} e^{f(x)-\left(\gamma, x-m^{*}\right)} \mu^{*}(d x) \rightarrow \inf _{\gamma \in D} .
$$

ОПРеДЕЛЕниЕ 2. Решением задачи (4) назовем вектор $\gamma^{*} \in D$ такой, что

$$
v \triangleq \inf _{\gamma \in D} \int_{E} e^{f(x)-\left(\gamma, x-m^{*}\right)} \mu^{*}(d x)=\int_{E} e^{f(x)-\left(\gamma^{*}, x-m^{*}\right)} \mu^{*}(d x) .
$$

4.2. Доказательство утверждений теоремы 1 опирается на разрешимость задачи (4). Нам понадобятся вспомогательные утверждения.

Предложение 2. Решение задачи (4) существует тогда и только тогда, когда выполняется неравенство (2).

ДокАЗАТЕЛЬСТво утверждения предложения 2 очевидно.

Справедливость утверждения 1) теоремы 1 вытекает из следующего предложения.

ПреДЛОЖенИЕ 3. Следующие утверждения эквивалентны:

1) $\gamma^{*}$ - решение задачи (4);

2) любая измеримая ограниченная функиия $f(x)$ допускает единственное представление

$$
f(x)=\int_{E} f(x) \mu^{*}(d x)+\left(\gamma^{*}, x-m^{*}\right) ;
$$

3) $\mu^{*}$ дискретна, причем $I^{\mu^{*}} \leqslant d+1$

ДокАЗАТЕЛЬСТво ПРЕДЛОЖЕНИЯ 3. ИМПликация из 1) в 2). Пусть $g: E \rightarrow \mathbb{R}^{+} \backslash\{0\}-$ любая $\mathscr{E}$-измеримая ограниченная функция. Положим для любого $A \in \mathscr{E}$

$$
\mu(A) \triangleq \int_{A} \frac{g(x)}{\int_{E} g(x) \mu^{*}(d x)} \mu^{*}(d x) .
$$

Ясно, что

i) $\mu \neq \mu^{*}$;

ii) $\mu \in \mathfrak{R}$.

Заметим, что мера $\mu^{*}$ доминирует любую меру $\widetilde{\mu} \in \mathfrak{R}$ и поэтому $\widetilde{\mu} \ll \mu^{*}$. Следовательно, справедливо неравенство

$$
v \triangleq \int_{E} e^{f(x)-\left(\gamma^{*}, x-m^{*}\right)} \mu^{*}(d x) \geqslant \int_{E} e^{f(x)-\left(\gamma^{*}, x-m^{*}\right)} \mu(d x),
$$

которое с учетом (7) можно переписать в виде

$$
\int_{E} g(x) \exp \left\{f(x)-\left(\gamma^{*}, x-m^{*}\right)-\ln v\right\} \mu^{*}(d x) \leqslant \int_{E} g(x) \mu^{*}(d x) .
$$


В силу произвольности измеримой функции $g(x)$ из (8) следует неравенство

$$
f(x)-\left(\gamma^{*}, x-m^{*}\right)-\ln v \leqslant 0 \quad \mu^{*} \text {-п.в. }
$$

С другой стороны, из (5) имеем равенство

$$
\int_{E} \exp \left\{f(x)-\left(\gamma^{*}, x-m^{*}\right)-\ln v\right\} \mu^{*}(d x)=1 .
$$

Следовательно, $\mu^{*}$-п.в.

$$
f(x)=\ln v+\left(\gamma^{*}, x-m^{*}\right) .
$$

Проинтегрируем обе части равенства (9) по мере $\mu^{*}$. Получим, что $\ln v=\int_{E} f(x) \mu^{*}(d x)$. Отсюда следует (6).

Докажем единственность представления (6). Доказательство проведем методом "от противного". Предположим, что существует $\widetilde{\gamma} \in D, \widetilde{\gamma} \neq \gamma^{*}$, такой, что $\mu^{*}$-п.в.

$$
f(x)=\int_{E} f(x) \mu^{*}(d x)+\left(\widetilde{\gamma}, x-m^{*}\right) .
$$

Вычтем из (10) равенство (6). Получим, что $\left(\widetilde{\gamma}-\gamma^{*}, x-m^{*}\right)=0 \mu^{*}$-п.в. Из ограниченности $f(x)$ и $(6)$ следует, что $\left(\gamma^{*}, x-m^{*}\right)^{2}$ ограничено. Поэтому относительно меры $\mu^{*}$ существует $K$ - ковариационная матрица случайного вектора $x$. Следовательно,

$$
\int_{E}\left(\widetilde{\gamma}-\gamma^{*}, x-m^{*}\right)^{2} \mu^{*}(d x)=\left(\widetilde{\gamma}-\gamma^{*}, K\left(\widetilde{\gamma}-\gamma^{*}\right)\right)=0 .
$$

Из положительной определенности матрицы $K$ и равенства (11) следует, что мы пришли к противоречию с предположением о том, что $\widetilde{\gamma} \neq \gamma^{*}$. Стало быть, наше предположение неверно и представление (6) единственно.

Импликация из 2) в 3). Сначала сделаем несколько замечаний. Поскольку $\mu^{*}-$ вероятностная мера, то она допускает единственное разложение (см. [12; §9]): $\mu^{*}=\alpha \mu^{* \mathrm{c}}+(1-\alpha) \mu^{* \mathrm{~d}}$, $\alpha \in[0,1]$, где $\mu^{* \mathrm{c}}$ - непрерывная вероятностная мера, $\mu^{* \mathrm{~d}}$ - дискретная вероятностная мера. Следовательно, мера $\mu^{* \mathrm{~d}}$ сосредоточена на атомах меры $\mu^{*}$, которых не более чем счетное число. Поскольку меры $\mu^{* \mathrm{~d}}$ и $\mu^{* \mathrm{c}}$ сингулярны $([12 ; \S 9])$, то существуют $\mathscr{E}$-измеримые множества $B$ и $\bar{B}$ такие, что:

i) $B \cup \bar{B}=E$;

ii) $B \cap \bar{B}=\varnothing$;

iii) $\mu^{* \mathrm{c}}(B)=1\left(\mu^{* \mathrm{~d}}(B)=0\right), \mu^{* \mathrm{~d}}(\bar{B})=1\left(\mu^{* \mathrm{c}}(\bar{B})=0\right)$.

Следовательно, для любого $A \in \mathscr{E}$ справедливы равенства

$$
\alpha \mu^{* \mathrm{c}}(A)=\mu^{*}(A \cap B), \quad(1-\alpha) \mu^{* \mathrm{~d}}(A)=\mu^{*}(A \cap \bar{B}),
$$

причем $\alpha=\mu^{*}(B), 1-\alpha=\mu^{*}(\bar{B})$.

Для доказательства этого утверждения достаточно показать, что для любого $A \in \mathscr{E}$ выполняется равенство

$$
\mu^{*}(A)=\mu^{* \mathrm{~d}}(A)
$$

В силу предположения индикаторы $1_{A}(x), 1_{\bar{B}}(x)$ и $1_{A \cap \bar{B}}(x)$ допускают соответственно единственные представления относительно меры $\mu^{*}$ :

$$
\begin{gathered}
1_{A}(x)=\mu^{*}(A)+\left(\gamma^{A}, x-m^{*}\right), \quad 1_{\bar{B}}(x)=\mu^{*}(\bar{B})+\left(\gamma^{\bar{B}}, x-m^{*}\right), \\
1_{A \cap \bar{B}}(x)=\mu^{*}(A \cap \bar{B})+\left(\gamma^{A \cap \bar{B}}, x-m^{*}\right) .
\end{gathered}
$$


Так как $1_{A}(x) 1_{\bar{B}}(x)=1_{A \cap \bar{B}}(x)$, то из $(14)$ и $(15)$ следует равенство $\mu^{*}$-п.в.

$$
\begin{aligned}
\mu^{*}(A \cap \bar{B})+\left(\gamma^{A \cap \bar{B}}, x-m^{*}\right)= & \mu^{*}(A) \mu^{*}(\bar{B})+\mu^{*}(A)\left(\gamma^{\bar{B}}, x-m^{*}\right) \\
& +\mu^{*}(\bar{B})\left(\gamma^{A}, x-m^{*}\right)+\left(\gamma^{A}, x-m^{*}\right)\left(\gamma^{\bar{B}}, x-m^{*}\right) .
\end{aligned}
$$

Равенство (16) выполняется для всех $x \in \operatorname{supp} \mu^{*}$. Так как $m^{*}-$ барицентр меры $\mu^{*}$, то он принадлежит относительной внутренности $\operatorname{supp} \mu^{*}$. Поэтому положим $x=m^{*}$. Тогда из (16) следует равенство $\mu^{*}(A \cap \bar{B})=\mu^{*}(A) \mu^{*}(\bar{B})$. Из этого равенства и (12) следует (13).

Для завершения доказательства этого утверждения осталось заметить, что

i) поскольку $\mu^{*}$ - дискретная вероятностная мера, то она является выпуклой комбинацией не более чем счетного числа мер Дирака $[11 ; \S 2$, гл.ІІІ], сосредоточенных в различных изолированных точках $x_{i} \in \operatorname{supp} \mu^{*} \subseteq E$;

ii) между мерами Дирака и точками $x_{i} \in \operatorname{supp} \mu^{*}$ существует взаимно однозначное соответствие;

iii) из теоремы Каратеодори $\left[13 ; \S 17\right.$, гл. IV] следует, что $I^{\mu^{*}} \leqslant d+1$.

Импликация из 3) в 1). Обозначим $\Phi(\gamma) \triangleq \int_{E} e^{f(x)-\left(\gamma, x-m^{*}\right)} \mu^{*}(d x)$. Поскольку мера $\mu^{*}$ дискретная и $I^{\mu^{*}} \leqslant d+1$, то $m^{*}$ и $\Phi(\gamma)$ допускают, соответственно, представления

$$
m^{*}=\sum_{i=1}^{I^{\mu^{*}}} c_{i} x_{i}, \quad \Phi(\gamma)=\sum_{i=1}^{I^{\mu^{*}}} c_{i} \exp \left\{f\left(x_{i}\right)-\left(\gamma, x_{i}-m^{*}\right)\right\}
$$

где $x_{i} \in \operatorname{supp} \mu^{*}$. Из условий теоремы также следует, что:

i) $D=\mathbb{R}^{d}$;

ii) для любого $x \in E$ справедливо неравенство $|f(x)| \leqslant c$.

Поэтому в силу неравенства Иенсена имеем неравенство

$$
\Phi(\gamma) \geqslant e^{-c}>0
$$

Перейдем к доказательству импликации. Пусть $I^{\mu^{*}}=1$. Тогда из (17) следует, что $m^{*}=x \in E$. Поэтому любое $\gamma \in \mathbb{R}^{d}$ является решением задачи (4).

Пусть $1<I^{\mu^{*}} \leqslant d+1$, а $\Gamma\left(\mu^{*}\right)-$ выпуклая оболочка supp $\mu^{*}$. Известно, что $m^{*}$ принадлежит относительной внутренности $\Gamma\left(\mu^{*}\right)$. Поэтому из $(17)$ следует, что существуют $x_{i}$, $x_{j} \in E$ такие, что $x_{i}<m^{*}, x_{j}>m^{*}$. Следовательно, $\Phi(\gamma)$-строго выпуклая, а в силу (18)ограниченная снизу функция, причем $\Phi(\gamma) \rightarrow \infty$ при $\gamma \rightarrow \infty$. Поэтому из (17) следует, что для любого ограниченного $\gamma \in D$ справедливы неравенства $\mu^{*}\left(\left(\gamma, x-m^{*}\right)>0\right)>0$ и $\mu^{*}\left(\left(\gamma, x-m^{*}\right)<0\right)>0$. Доказательство закончено.

ЗАмЕчАниЕ 3. Из доказательства импликации из 2) в 3) предложения 3 и единственности представления (6) следует единственность дискретной меры $\mu^{*}$.

4.3. ДоКАЗАТЕЛЬСтво ПУНКтА 2) теоремы 1. Из компактности множества $E$ следует, что:

i) $\mathfrak{R}=M(E)$ - компакт в топологии слабой сходимости вероятностных мер;

ii) в силу предложения 1 существует вероятностная мера $\mu^{*} \in M(E)$, которая доминирует любую другую меру $\mu \in M(E)$, следовательно, $\mu \ll \mu^{*}$ и поэтому $\mu^{*}-$ крайняя точка множества $M(E)$;

iii) существует $\gamma^{*} \in D$, для которого справедливо (2). 
Поэтому из утверждения предложения 3 следует, что мера $\mu^{*}$ дискретна. Из дискретности меры $\mu^{*}$ следует, что она является выпуклой оболочкой мер Дирака. Как уже отмечалось при доказательстве импликации из 2) в 3) предложения 3, между мерами Дирака и точками $x_{i} \in \operatorname{supp} \mu^{*}$ существует взаимнооднозначное соответствие. Поэтому в силу теоремы Каратеодори мера $\mu^{*}$ сосредоточена не более чем в $d+1$ точке. Поскольку мера $\mu^{*}$ крайняя, то ее носитель сосредоточен на extr $E$. Из вышесказанного следует справедливость (3).

ЗАмечание 4 . Если $f(x)$ - непрерывная функция, то утверждение пункта 2) теоремы 1 известно. В этом случае интерес представляет новое доказательство этого утверждения.

ЗАмечание 5. Из утверждения пункта 2) теоремы 1 следует, что носитель меры $\mu^{*}$ сосредоточен на extr $E$. Легко убедиться в том, что справедливо утверждение, обратное утверждению пункта 2) теоремы 1.

\section{СПИСОК ЦИТИРОВАННОЙ ЛИТЕРАТУРЫ}

[1] Р. Фелпс, Лекиии о теоремах Шоке, Мир, М., 1968. [2] П.А. Мейер, Вероятность и потенииаль, Мир, М., 1973. [3] R. D. Bourgin, Geometric Aspects of Convex Sets with the Radon-Nikodým Property, Lecture Notes in Math., 993, Springer-Verlag, Berlin, 1983. [4] R. D. Bourgin, G. A. Edgar, J. Functional Analysis, 23:2 (1976), 162-176. [5] G. A. Edgar, J. Functional Analysis, 23:2 (1976), 145-161. [6] P. Mankiewicz, Studia Math., 63:3 (1978), 259-265. [7] А. Н. Ширяев, Основы стохастической финансовой математики. Т. 2. Теория, ФАЗИС, М., 1998. [8] Г. Фельмер, А. Шид, Введение в стохастические финансы. Дискретное время, МЦНМО, М., 2008. [9] О. В. Зверев, В. М. Хаметов, Обозр. прикл. и промыш. матем., 18:1 (2011), 26-54. [10] В. И. Богачев, А. В. Колесников, УМН, 67:5(407) (2012), 3-110. [11] А. Н. Ширяев, Вероятность-1, МЦНМО, М., 2004. [12] Г. Е. Шилов, Б. Л. Гуревич, Интеграл, мера и производная. Общая теория, Наука, М., 1967. [13] Р. Рокафеллар, Выпуклый анализ, Мир, М., 1973.

\section{Г. А. Васильев}

Поступило

Московский государственный

институт электроники и математики -

Высшая школа экономики

E-mail: math@glebvasiliev.ru

\section{В. М. Хаметов}

Московский государственный

институт электроники и математики -

Высшая школа экономики

E-mail: khametovvm@mail.ru

\section{Е. А. Шелемех}

Московский государственный институт электроники и математики Высшая школа экономики

E-mail: letis@mail.ru 